\title{
Cardiotoxicity of antiangiogenic drugs: causes and mechanisms
}

\section{Beata Tomaszewska1, Małgorzata Muzolf', Radosław Grabysa', 3 , Lubomir Bodnar ${ }^{4}$}

'Department of Oncology and Immuno-Oncology, Warmian-Masurian Cancer Center of the Ministry of the Interior and Administration's Hospital, Olsztyn, Poland

${ }^{2}$ Department of Internal Medicine, Warmian-Masurian Cancer Center of the Ministry of the Interior and Administration's Hospital, Olsztyn, Poland

${ }^{3}$ Department of Pulmonology, Collegium Medicum, University of Warmia and Mazury, Olsztyn, Poland

${ }^{4}$ Department of Oncology and Immuno-Oncology, Collegium Medicum, University of Warmia and Mazury, Olsztyn, Poland

Correspondence: Beata Anna Tomaszewska Department of Oncology, Warmian-Masurian Cancer Center of the Ministry of the Interior and Administration's Hospital 10-228 Olsztyn, Poland, Wojska Polskiego 37 tel.: +48895398511 e-mail:beata.kosinska@gmail.com

Received: 8.02.2021 Accepted: 8.03.2021

DOI: 10.24292/01.OR.122030321 Copyright ( ) Medical Education. All rights reserved.

\section{ABSTRACT}

Therapy with angiogenesis inhibitors is undoubtedly an advancement in cancer treatment; however, it is associated with a risk of developing cardiotoxicity, which most often manifests in myocardial contractile dysfunction or an increased risk of thromboembolic events. Heart failure is observed in $2-4 \%$ of patients treated with bevacizumab and in 3-8\% of patients on antiangiogenic tyrosine kinase inhibitors.

The proposed pathomechanisms underlying the impairment in systolic function during antiangiogenic drug treatment include mitochondrial dysfunction, a secondary reduction in cardiomyocyte ATP production and redox imbalance, which may contribute to pathological states known as "free radical diseases". Additionally, therapy with angiogenesis inhibitors may also cause cardiac oxidative stress.

The risk factors for cardiac complications include arterial hypertension, which is a known "class effect" of this class of drugs, as well as a number of other factors such as age, comorbidities, prior radiotherapy and baseline left ventricular ejection fraction.

The cardiovascular diseases are still the first cause of death in the world. The more effective oncological treatment becomes, the more often comorbidities occur. This fact seems to demand interdisciplinary approach from investigators and practitioners.

This article presents the current state of knowledge on the molecular mechanisms of cardiotoxicity of antiangiogenic drugs used in routine clinical practice.

Key words: antiangiogenic agents, bevacizumab, cardiooncology, sorafenib, sunitinib 


\section{INTRODUCTION}

Cardiac toxicity is a broad term that encompasses the most clinically significant complications of cancer therapy. The term "cardiotoxicity" is generally used to describe all cardiac adverse effects caused by oncological treatment or by specific cardiovascular disorders, such as hypertension or hypotension, left ventricular systolic dysfunction, myocardial infarction, rhythm and conduction disturbances, myocarditis and pericarditis, and in the case of radiotherapy, coronary artery disease, valve defects and pericardial complications, which may occur during such therapy [1]. The practice guidelines of the Task Force of the European Society of Cardiology under the heading cardiovascular complications in cancer treatment also include issues related to the risk of lipid disorders, stroke and pulmonary hypertension, as well as thromboembolic events [2].

In addition to regular electrocardiographic evaluation, the "gold standard" for cardiotoxicity assessment is echocardiographic evaluation of left ventricular ejection fraction (LVEF). Modern diagnostic methods make it possible to assess the direct effect of anticancer treatment on the structure of the heart (e.g., fibrosis), rhythm and conduction disturbances, diastolic function, function of systemic and pulmonary blood vessels, haemodynamics, haemostasis and the heart's response to stress factors. Changes in myocardial strain $(\varepsilon)$ and the strain rate (SR) observed in colour-coded tissue Doppler images during cancer treatment, as well as specific cardiovascular biomarkers (e.g., natriuretic peptide type $B$ and cardiac troponins), may illustrate subtle disturbances in the cardiovascular system, appearing at a very early stage of heart damage, much earlier than the decrease in LVEF [3].

Most cancerous tumours cannot grow beyond $2-3 \mathrm{~mm}^{3}$ without forming their own network of blood vessels (angiogenesis). This is due to the need to provide oxygen to the growing cancerous tissue. Discovery of the tumour's ability to create and develop its own vascularity, and attempts to inhibit it, have become the basis for the development of new cancer therapy strategies [4]. The most efficient molecules with antiangiogenic potential are those that directly block the activity of vascular endothelial growth factor type A (VEGF-A) and its VEGFR-1 and VEGFR-2 receptors. The above receptors can be inactivated by specific antibodies and by small molecule tyrosine kinase inhibitors (TKIs). The first group of antibodies that directly neutralise VEGF include bevacizumab, ramucirumab and aflibercept. Those that work via a mechanism of blocking VEGFR-1 and VEGFR-2 receptors include sunitinib, sorafenib, pazopanib, axitinib, regorafenib, nintedanib and cabozantinib [5]. In addition to their unquestionable antitumour effi- cacy, these drugs also cause a wide range of cardiovascular complications. These are mainly arterial hypertension, left ventricular systolic dysfunction and the risk of thromboembolic complications. Cardiac arrhythmias and acute coronary events, including sudden cardiac death, are observed much less frequently.

\section{MOLECULAR MECHANISMS UNDERLYING THE CARDIOTOXICITY OF ANTIANGIOGENIC THERAPIES}

\section{Arterial hypertension}

Blood pressure may rise in the first days of antiangiogenic therapy. In the case of sorafenib, it has been estimated that this effect may occur even before therapeutic drug levels are achieved. From this, it can be concluded that the primary mechanism for the increase in blood pressure is blocking the production of endothelial vasodilators such as nitric oxide (NO), and perhaps others like haem oxidase, endothelin 1 or adrenomedullin [6].

When explaining the pathomechanism for this phenomenon, the role of hypoxia should first of all be emphasised. Under physiological conditions, VEGF synthesis can be triggered, among other factors, by tissue hypoxia. When the cell becomes depleted of oxygen, the expression of hypoxia-induced factor (HIF), a potent transcription factor for VEGF, is increased. This, in turn, stimulates the mitosis of endothelial cells and their migration to vascular regions of the hypoxic area. Furthermore, by stimulating endothelial nitric oxide synthetase, it also stimulates endothelial cells to produce NO [6].

Direct VEGF administration may induce vasodilation and severe hypotension in patients. This is due to the fact that increased expression of endothelial nitric oxide synthase through the phosphoinositide 3-kinase/AKT (PI3K/AKT) and mitogen-activated protein kinase (MAPK) - dependent pathways leads to increase NO production and, consequently, vasodilation. Most of the increases in blood pressure are observed in the first week of sorafenib therapy and normalise soon after treatment is stopped, which may explain the hypothesis that endothelial-mediated vasoconstriction is the cause of most of the observed increases in blood pressure [7].

Preclinical studies and observations in patients have shown that endothelial cell apoptosis, which leads to a reduction in blood vessel density and an increase in afterload, may also play an important role in the pathogenesis of arterial hypertension. It needs to be highlighted that VEGF is a factor that strongly stimulates the proliferation of endothelial cells. In an animal model (mouse 
xenografts with implanted kidney cancer tumours), a reduction in endothelial cells in the tumour vessels was observed as early as on the third day of treatment with a VEGF inhibitor. VEGF inhibitors induce apoptosis of endothelial cells and a decrease in capillary density. These changes were seen in vessels in skin biopsies of patients treated with sorafenib. Moreover, necrosis of the basal membrane of capillaries could also be observed, indicating that endothelial cell apoptosis under anti-VEGF therapy is not selective for tumour vascularisation [8].

\section{Left ventricular systolic dysfunction}

The pathomechanisms underlying the deterioration in left systolic ventricular systolic function are the subject of intensive research. There are reports of cardiomyocyte hypertrophy and changes in the structure of mitochondria (without signs of in flammation and fibrosis) that are visible under electron microscopy of myocardial biopsies of patients treated with sunitinib [9]. However, when analysing the above reports, it should be taken into account that these patients had previously been exposed to the cardiotoxic effects of other drugs, which could potentially generate visible structural changes in cardiomyocytes.

Among the proposed pathomechanisms of impaired left ventricular systolic function, the most likely is mitochondrial dysfunction with a secondary reduction in cardiomyocyte ATP production. Analysis of an animal model (mitochondria isolated from rat myocardium cells) showed negative changes in mitochondrial function after the administration of dasatinib, imatinib, sunitinib and sorafenib, with the greatest toxicity observed with sorafenib [10]. Sunitinib may cause heart failure, cardiomyopathy and left ventricular dysfunction, which may be attributed to its initiation of mitochondrial dysfunction. Mitochondrial dysfunction and redox imbalance may contribute to pathological states known as "free radical diseases". In an animal model, sunitinib significantly decreased glutathione and glutathione reductase activity and increased malondialdehyde, an indicator of lipid peroxidation in cardiac tissues [11].

Blockade of RAF1 and BRAF kinases appears to be the leading pathomechanism underlying the cardiac complications of sorafenib treatment. In experimental studies in animal models, blocking RAF1 has been demonstrated to cause heart wall hypokinesis and lead to heart dilatation, with greatly increased apoptosis and cardiomyocyte fibrosis. These disorders were significantly more pronounced with the coexistence of cardiac pressure overload. It can therefore be concluded that the effect of iatrogenic arterial hypertension induction may play a role in the pathogenesis of cardiac complications of sorafenib. It must be noted that VEGF plays a key role in both angiogenesis and vasculogenesis. Research suggests that VEGF is an essential factor for maintaining myocardial capillary density, and a reduction in the vascular bed (which is a negative effect of anti-VEGF therapy) is associated with the development of compensatory myocardial hypertrophy leading to heart failure [12].

When the VEGF-VEGFR pathway is blocked, the density of capillaries in the heart muscle is reduced, contractility is impaired and fibrosis develops, but these processes only occur under pressure overload conditions. Another important potential mechanism is blocking of the platelet-derived growth factor receptor (PDGFR), which determines the survival of cardiomyocytes subjected to haemodynamic stress (a study in a mouse model showed an increase in PDGFR- $\beta$ expression in myocardial cells in response to cardiac overload) [13].

Another experimental study in an animal model found no markers of apoptosis or fibrosis in the cardiomyocytes of mice that were not subjected to pharmacological induction of hypertension after sunitinib administration. The observed phenomenon may support the hypothesis that the cardiotoxicity of angiogenesis inhibitors may be dependent on the coexistence of iatrogenic arterial hypertension, which is a typical complication of this class of drugs [14].

During the evaluation of mechanisms underlying the negative consequences of sunitinib use, it was shown that mitochondrial proapoptotic pathways, activated by the release of cytochrome C into the cytosol and the activation of caspase 9, play an important role. Blocking the KIT gene pathway is another postulated mechanism for the cardiotoxicity of sunitinib. The membrane KIT receptor, the ligand of which is stem cell factor (SCF), is blocked by sunitinib and sorafenib, as well as by imatinib, nilotinib and dasatinib. The KIT receptor is present on haemangioblasts, which are precursors to hematopoietic stem cells and endothelial progenitor cells (EPCs). The normal function of the KIT receptor appears to be essential for the mobilisation of EPCs in lesions such as ischemic foci. Blocking this pathway could therefore lead to the dysfunction of endothelial cells and impair their repair mechanisms. Electron microscopy of myocardial cells from laboratory animals treated with sunitinib revealed mitochondrial oedema, changes in cytoplasm density and vacuolisation, as well as adverse changes in mitochondrial membrane potentials [15] . Damage to the mitochondrial reticulum has been reported in animals receiving sorafenib, which was not seen with pazopanib. In the cardiomyocytes of animals receiving sunitinib or sorafenib, significant reductions in ATP stocks were observed, which was not 
seen with pazopanib. These studies have shown that this adverse effect on the heart muscle is not a common effect of antiangiogenic drugs, but that it is dependent on the extent of tyrosine kinases blocked by individual preparations [16].

There are conflicting published data on the reversibility of angiogenesis inhibitor-induced cardiac dysfunction; either denying its reversibility $[17,18]$ or confirming the existence of such a possibility $[19,20]$. Oncologists observe in patients not only a decrease in LVEF correlating with systolic dysfunction of the left ventricle but also increases in myocardial necrosis markers. Among cancer patients clinical practicioners observe not only a decrease in LVEF correlating with systolic dysfunction of the left ventricle. There are increases in myocardial necrosis markers such as cardiac troponins, non-specific changes in electrocardiogram (ECG) and increases in N-terminal prohormone of brain natriuretic peptide (NT-proBNP) levels. Reports have confirmed that the introduction of optimal cardiac treatment leads to the normalisation of these parameters [21]. Some researchers postulate that cardiomyocyte necrosis does not occur through the mechanism of apoptosis, but is a consequence of unfavourable energy metabolism and an overload of cardiomyocytes with lipids [16].

\section{MECHANISM OF TOXIC ACTION RELATED TO THE RISK OF THROMBOEMBOLISM}

A model example of an antiangiogenic drug proven to have the greatest impact on the risk of venous thromboembolism (VTE) is bevacizumab. The possible cardiac side effects of treatment with this drug also include arterial hypertension and bleeding complications.
Bevacizumab is a recombinant humanised monoclonal lgG antibody. By specifically binding to VEGF, it inhibits the process of neoangiogenesis by blocking the binding of VEGF to a membrane receptor. Among the unfavourable effects of this drug, a reduction in the anti-inflammatory effect of VEGF has been reported, leading to increased inflammation and instability of atherosclerotic plaques, resulting in damage to the plaque and formation of a clot. The increased risk of thromboembolic events is correlated to the degree of endothelial damage, as evidenced by an increase in von Willebrand factor, E-selectin and tissue factor levels [22]. As previously mentioned, VEGF is an important factor in promoting and repairing endothelial cells. Thus, anti-VEGF therapy can reduce the regenerative capacity of endothelial cells in response to damage and expose the subendothelial collagen. In addition, VEGF is indirectly involved in the secretion of prostaglandin (PGI2) and NO by the endothelium [15]. Anti-VEGF therapy reduces the amount of $\mathrm{NO}$ and prostacyclins and also increases blood viscosity by overproducing erythropoietin, leading to an increased risk of thromboembolic events [23].

\section{OTHER CARDIAC COMPLICATIONS}

Each of the above side effects is also a significant risk factor for one of the most serious events, potentially fatal acute coronary syndrome (ACS). This complication is most often associated with TKI therapy and is a consequence of the above-mentioned molecular mechanisms (tab. 1). Symptoms of ACSs (including myocardial infarction) appear significantly more frequently in patients treated with sorafenib compared to a placebo group, observed in $2.9 \%$ and $0.4 \%$ of patients, respectively [24]. The risks of infarction or cardiac ischemia associated with other angi-

TABLE 1.

Frequency of the most common cardiotoxicities of antiangiogenic drugs.

\begin{tabular}{|c|c|c|c|c|}
\hline Drug & Main molecular targets & $\begin{array}{c}\text { The most common cardiovascular } \\
\text { toxicities }\end{array}$ & Frequency (\%) & References \\
\hline sunitinib & VEGFR, PDGFR, KIT, FIt3, CSF-1R, RET & $\begin{array}{l}\text { Hypertension } \\
\text { LVEF decrease } \\
\text { Venous thrombosis }\end{array}$ & $\begin{array}{l}36 \% \\
4 \% \\
3 \%\end{array}$ & Ravaud, 2016 [25] \\
\hline pazopanib & VEGFR, PDGFR, C-KIT & $\begin{array}{c}\text { Thromboembolic events } \\
\text { Hypertension }\end{array}$ & $\begin{array}{l}4 \% \\
40 \%\end{array}$ & Justice, 2018 [26] \\
\hline vandetanib & EGFR, VEGFR, RET & $\begin{array}{c}\text { QT interval prolongation over } \\
500 \mathrm{~ms} \\
\text { Hypertension }\end{array}$ & $\begin{array}{l}13 \% \\
24 \%\end{array}$ & Grande, 2013 [27] \\
\hline bevacizumab & VEGF-A & $\begin{array}{c}\text { Hypertension } \\
\text { Arterial thrombosis }\end{array}$ & $\begin{array}{l}42 \% \\
3,8 \%\end{array}$ & Bonaldo, $2020[28]$ \\
\hline cabozantinib & $\begin{array}{c}\text { VEGFR, MET, AXL, KIT, ROS1, RET, } \\
\text { TIE2,FIt3 }\end{array}$ & Hypertension grade 3 & $28 \%$ & Choueiri, $2018[29]$ \\
\hline ramucirumab & VEGFR2 & Hypertension grade 3 & $14 \%$ & Wilke, 2014 [30] \\
\hline lenvatinib & VEGFR, FGFR, PDGFR, Kit, RET & Hypertension & $42 \%$ & Kudo, 2018 [31] \\
\hline aksytynib & VEGFR, PDGFR, c-KIT & Hypertension & $40 \%$ & Rini, 2011 [32] \\
\hline nintedanib & FGFR, PDGFR, VEGFR & $\begin{array}{l}\text { Chest pain (docetaxel plus } \\
\text { nintedanib) }\end{array}$ & $8 \%$ & Reck, 2014 [33] \\
\hline
\end{tabular}


ogenesis inhibitors are very low $(1.5 \%$ for bevacizumab, $<1 \%$ for sunitinib) [34]. However, reports suggest that following successful interventional treatment of such incidents, patients can safely continue TKI therapy [35]. In an evaluative meta-analysis of the frequency of myocardial ischemia (MI) in patients treated with TKIs, the authors found that $\mathrm{MI}$ can potentially develop in 1 out of every 85 patients treated with VEGF inhibitors, but these data were based on an analysis of only eight studies. Importantly, the risk of fatal cardiac events is low, amounting to $0.25 \%$, meaning that one out of every 1259 treated patients will experience a fatal event [36].

Based on reports on the occurrence of ventricular arrhythmias and sudden cardiac death events during therapy with TKls, a hypothesis was made about the possible effect of these drugs on prolongation of the QT interval in ECG as a cause of fatal cardiac complications. Ventricular arrhythmias, particularly torsades de pointes (TdP), correlate with QTc prolongation above $500 \mathrm{~ms}$. A meta-analysis involving 18 clinical trials with a total of 6548 patients treated with kinase inhibitors showed a significant increase in the risk of QTc prolongation of each grade in anti-VEGFR-treated patients compared to patients not treated with TKIs, which was 8.66 times higher (95\% Cl [4.92-15.2]; $p<0.001)$. The risk of QTc prolongation above $500 \mathrm{~ms}$ was also significantly increased in anti-VEGFR-treated patients ( $\mathrm{RR}=2.69 ; 95 \% \mathrm{Cl}[1.33-5.44]$; $p=0.006$ ). Longer term therapy did not increase the relative risk of this complication. It can therefore be concluded that the risk is constant over time and that early detection plays a key role in preventing fatal events. A study by Bello et al. [37] assessed the pharmacokinetics of sunitinib in terms of QT prolongation. It was shown that the time to record the greatest QTc increase did not correlate with the peak drug concentration, which would suggest a delayed QT prolongation. There are no data in the literature on the correlation between the length of drug exposure and the effect on QT prolongation [38].

The postulated mechanism for drug-induced QT prolongation involves the direct influence of the three-dimensional molecular structure of the drug reacting with the hERG K+ myocardial potassium channels, resulting in impaired depolarisation wave flow and delayed impulse conduction [39]. Preclinical studies with sunitinib and vandetanib have shown that these drugs react with hERG potassium channels. Another hypothesised mechanism for QTC prolongation is inhibition of the sorting of proteins associated with potassium channels. Interference in the process of leaving the endoplasmic reticulum by the chaperones of hERG channels leads to a reduction in flow through the potassium channels. This is potentially the result of misfolding of proteins due to the action of specific drugs or from modification of interactions of chaperones with other proteins (fig. 1) [40, 41].

Baseline cardiovascular health and electrolyte disturbances also contribute to drug-induced QT prolongation. Treatment accompanying anticancer therapy with the potential for QT prolongation may have an additive negative impact on the risk of conduction disturbances. Additionally, the influence of supportive treatment commonly used in oncology, such as ondansetron, palonosetron, granisetron, prochlorperazine and olanzapine, as well as painkillers (e.g., methadone) and antidepressants (e.g., citalopram, escitalopram, venlafaxine, sertraline and mirtazapine), are particularly important as they can make these disorders worse [38].

FIGURE 1.

Mechanisms of QT interval prolongation. direct mechanism

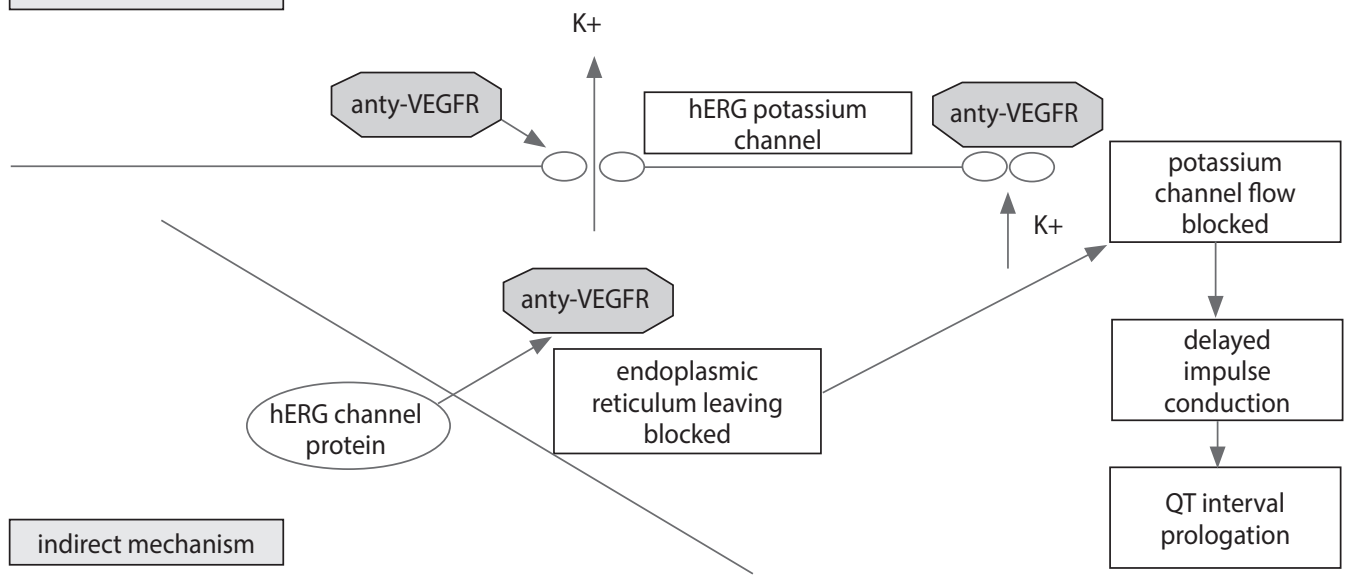




\section{CONCLUSIONS}

The effectiveness of therapy with drugs with antiangiogenic potential has prolonged the overall survival and improved disease control of some cancers. However, the use of antiangiogenic therapies is associated with an increased risk of cardiotoxicity. When analysing the results of clinical trials, it can be assumed that the number of cardiac complications is underestimated. Patients recruited for trials are often younger and less burdened with comorbidities than the general population. Despite the constant advancements in cardiological diagnostic methods and their widespread availability, recognition of the predictors of cardiotoxicity of VEGF inhibitors and the possibility of their detection and possible early elimination remains in the research phase and requires further in-depth observations, especially prospective trials.

\section{References}

1. Lenneman CG, Sawyer DB. Cardio-Oncology: An Update on Cardiotoxicity of Cancer-Related Treatment. Circ Res. 2016; 118(6): 1008-20

2. Zamorano JL, Lancellotti P, Rodriguez Muńoz D et al. 2016 ESC Position Paper on cancer treatments and cardiovascular toxicity developed under the auspices of the ESC Committee for Practice Guidelines: The Task Force for cancer treatments and cardiovascular toxicity of the European Society of Cardiology (ESC). Eur Heart J. 2016; 37(36): 2768-801.

3. Sawaya H, Sebag IA, Plana JC et al. Early detection and prediction of cardiotoxicity in chemotherapy-treated patients. Am J Cardiol. 2011; 107(9): 1375-80.

4. Wilson WR, Hay MP. Targeting hypoxia in cancer therapy. Nat Rev Cancer. 2011; 11(6): 393-410.

5. Ferrara N, Adamis AP. Ten years of anti-vascular endothelial growth factor therapy. Nat Rev Drug Discov. 2016; 15(6): 385-403.

6. Rey S, Schito L, Wouters BG et al. Targeting Hypoxia-Inducible Factors for Antiangiogenic Cancer Therapy. Trends Cancer. 2017; 3(7): 529-41.

7. Eppler SM, Combs DL, Henry TD et al. A target-mediated model to describe the pharmacokinetics and hemodynamic effects of recombinant human vascular endothelial growth factor in humans. Clin Pharmacol Ther. 2002; 72(1): 20-32.

8. Humphreys BD, Atkins MB. Rapid development of hypertension by sorafenib: toxicity or target? Clin Cancer Res. 2009; 15(19): 5947-9.

9. Chu TF, Rupnick MA, Kerkela R et al. Cardiotoxicity associated with tyrosine kinase inhibitor sunitinib. Lancet Lond Engl. 2007; 370(9604): 2011-9.

10. Will Y, Dykens JA, Nadanaciva $S$ et al. Effect of the multitargeted tyrosine kinase inhibitors imatinib, dasatinib, sunitinib, and sorafenib on mitochondrial function in isolated rat heart mitochondria and H9c2 cells. Toxicol Sci. 2008; 106(1): 153-61.

11. Imam F, Al-Harbi NO, Khan MR et al. Protective Effect of RIVA Against Sunitinib-Induced Cardiotoxicity by Inhibiting Oxidative Stress-Mediated Inflammation: Probable Role of TGF- $\beta$ and Smad Signaling. Cardiovasc Toxicol. 2020; 20(3): 281-90.

12. Izumiya Y, Shiojima I, Sato K et al. Vascular endothelial growth factor blockade promotes the transition from compensatory cardiac hypertrophy to failure in response to pressure overload. Hypertens Dallas Tex. 1979. 2006; 47(5): 887-93.

13. Chintalgattu V, Ai D, Langley RR et al. Cardiomyocyte PDGFR-beta signaling is an essential component of the mouse cardiac response to load-induced stress. J Clin Invest. 2010; 120(2): 472-84.

14. Gupta R, Maitland ML. Sunitinib, hypertension, and heart failure: a model for kinase inhibitor-mediated cardiotoxicity. Curr Hypertens Rep. 2011; 13(6): 430-5.

15. Szmit S. The cardiac safety during treatment with sunitinib and sorafenib, multikinase angiogenesis inhibitors. OncoReview. 2012; $2(6): 134-42$.

16. Force T, Krause DS, Van Etten RA. Molecular mechanisms of cardiotoxicity of tyrosine kinase inhibition. Nat Rev Cancer. 2007; 7(5): 332-44.

17. Khakoo AY, Kassiotis $\mathrm{CM}$, Tannir $\mathrm{N}$ et al. Heart failure associated with sunitinib malate: a multitargeted receptor tyrosine kinase inhibitor. Cancer. 2008; 112(11): 2500-8.

18. Garcipérez de Vargas FJ, Gómez-Barrado JJ, Ortiz C et al. [Refractory heart failure in a patient treated with bevacizumab]. Med Intensiva. 2012; 36(8): 589-90.

19. Hawkes EA, Okines AFC, Plummer C et al. Cardiotoxicity in patients treated with bevacizumab is potentially reversible. J Clin Oncol. 2011; 29(18): e560-2.

20. Szmit S, Nurzyński P, Szaluś $N$ et al. Reversible myocardial dysfunction in a young woman with metastatic renal cell carcinoma treated with sunitinib. Acta Oncol Stockh Swed. 2009; 48(6): $921-5$.

21. Schmidinger M, Zielinski CC, Vogl UM et al. Cardiac toxicity of sunitinib and sorafenib in patients with metastatic renal cell carcinoma. J Clin Oncol. 2008; 26(32): 5204-12.

22. Kuenen BC, Levi M, Meijers JCM et al. Analysis of coagulation cascade and endothelial cell activation during inhibition of vascular endothelial growth factor/vascular endothelial growth factor receptor pathway in cancer patients. Arterioscler Thromb Vasc Biol. 2002; 22(9): 1500-5.

23. Economopoulou P, Kotsakis A, Kapiris I et al. Cancer therapy and cardiovascular risk: focus on bevacizumab. Cancer Manag Res. 2015; 7: 133-43.

24. Escudier B, Eisen T, Stadler WM et al. Sorafenib in advanced clear-cell renal-cell carcinoma. N Engl J Med. 2007; 356(2): 125-34.

25. Ravaud A, Motzer RJ, Pandha HS et al; S-TRAC Investigators. Adjuvant Sunitinib in High-Risk Renal-Cell Carcinoma after Nephrectomy. N Engl J Med. 2016; 375(23): 2246-54

26. Justice CN, Derbala MH, Baich TM et al. The Impact of Pazopanib on the Cardiovascular System. J Cardiovasc Pharmacol Ther. 2018; 23(5): 387-98.

27. Grande E, KreissI MC, Filetti $S$ et al. Vandetanib in advanced medullary thyroid cancer: review of adverse event management strategies. Adv Ther. 2013; 30(11): 945-66.

28. Bonaldo G, Vaccheri A, Melis M, Motola D. Drug-induced disseminated intravascular coagulation: a pharmacovigilance study on World Health Organization's database. J Thromb Thrombolysis. 2020; 50(4): 763-71.

29. Choueiri TK, Hessel C, Halabi S et al. Cabozantinib versus sunitinib as initial therapy for metastatic renal cell carcinoma of intermediate or poor risk (Alliance A031203 CABOSUN randomised trial): Progression-free survival by independent review and overall survival update. Eur J Cancer. 2018; 94: 115-25.

30. Wilke H, Muro K, Van Cutsem E et al; RAINBOW Study Group. Ramucirumab plus paclitaxel versus placebo plus paclitaxel in patients with previously treated advanced gastric or gastro-oesophageal junction adenocarcinoma (RAINBOW): a double-blind, randomised phase 3 trial. Lancet Oncol. 2014; 15(11): 1224-35.

31. Kudo $\mathrm{M}$, Finn RS, Qin $\mathrm{S}$ et al. Lenvatinib versus sorafenib in first-line treatment of patients with unresectable hepatocellular carcinoma: a randomised phase 3 non-inferiority trial. Lancet. 2018; 391(10126): 1163-73.

32. Rini BI, Escudier B, Tomczak $P$ et al. Comparative effectiveness of axitinib versus sorafenib in advanced renal cell carcinoma (AXIS): a randomised phase 3 trial. Lancet. 2011; 378(9807): 1931-9. 
33. Reck M, Kaiser R, Mellemgaard A; LUME-Lung 1 Study Group. Docetaxel plus nintedanib versus docetaxel plus placebo in patients with previously treated non-small-cell lung cancer (LUME-Lung 1): a phase 3, double-blind, randomised controlled trial. Lancet Oncol. 2014; 15(2): 143-55.

34. Pantaleo MA, Mandrioli A, Saponara $\mathrm{M}$ et al. Development of coronary artery stenosis in a patient with metastatic renal cell carcinoma treated with sorafenib. BMC Cancer. 2012; 12: 231

35. Habib M, Czernek U, Dębska S et al. Ostry zespół wieńcowy w trakcie leczenia sorafenibem - opis chorej. Oncol Clin Pract. 2012; 8(3): 124-7.

36. Abdel-Qadir H, Ethier J-L, Lee DS et al. Cardiovascular toxicity of angiogenesis inhibitors in treatment of malignancy: A systematic review and meta-analysis. Cancer Treat Rev. 2017; 53: 120-7.

37. Bello CL, Mulay $M$, Huang $X$ et al. Electrocardiographic characterization of the QTC interval in patients with advanced solid tumors: pharmacokinetic - pharmacodynamic evaluation of sunitinib. Clin Cancer Res. 2009; 15(22): 7045-52.

38. Ghatalia P, Je Y, Kaymakcalan MD et al. QTc interval prolongation with vascular endothelial growth factor receptor tyrosine kinase inhibitors. Br J Cancer. 2015; 112(2): 296-305.

39. Sanguinetti MC, Mitcheson JS. Predicting drug-hERG channel interactions that cause acquired long QT syndrome. Trends Pharmacol Sci. 2005; 26(3): 119-24.

40. Obers S, Staudacher I, Ficker E et al. Multiple mechanisms of hERG liability: K+ current inhibition, disruption of protein trafficking, and apoptosis induced by amoxapine. Naunyn Schmiedebergs Arch Pharmacol. 2010; 381(5): 385-400.

41. Dennis AT, Wang L, Wan $\mathrm{H}$ et al. Molecular determinants of pentamidine-induced hERG trafficking inhibition. Mol Pharmacol. 2012; 81 (2): 198-209.

Authors' contributions:

Beata Tomaszewska: conceptualization, writing of original draft preparation, revised the manuscript; Małgorzata Muzolf: critical review of the manuscript, revised the manuscript; Radosław Grabysa: critical review of the manuscript, revised the manuscript; Lubomir Bodnar: conceptualization, writing of original draft preparation, supervised the wrote the manuscript, revised the manuscript.

Conflict of interests: Authors declare no conflict of interest.

Financial support: There was no financial support. 